\title{
Ulceration of the ileum in Crohn's disease: correlation with vascular anatomy
}

\author{
Andrew Anthony, Amar P Dhillon, Roy E Pounder, Andrew J Wakefield
}

\begin{abstract}
Background-Ileal ulcers in Crohn's disease tend to lie along the same side of the bowel wall as the mesenteric attachment; the mesenteric and antimesenteric borders are supplied by short and long arteries, respectively.

Aim-To examine the localisation of ileal Crohn's ulcers and to test the hypothesis that predilection of Crohn's ulcers for the ileal mesenteric margin is explained by the existence of end arteries that supply the mesenteric margin.

Methods-The localisation of ulcers in the bowel wall was examined in eight resection specimens of Crohn's disease of the terminal ileum. The vascular anatomy of normal terminal ileum $(n=8)$ and proximal jejunum $(n=8)$ postmortem specimens was studied; isolated long and short vessels were ligated before perfusion in four of these specimens.
\end{abstract}

Results-All eight specimens of Crohn's disease of the terminal ileum showed longitudinal ulceration along the mesenteric margin. In the postmortem study, the submucosal vascular plexus derived from ileal, but not jejunal short vessels, comprised end arteries with little or no communication with the submucosal plexus arising from long vessels. Prior ligation of ileal, but not jejunal, short vessels resulted in a filling defect of the submucosal plexus along the mesenteric margin in three of the four specimens. Ligation of ileal and jejunal long vessels did not affect carbon ink perfusion of the bowel wall.

Conclusions-In the human terminal ileum, the short vessels supplying the mesenteric margin are end arteries, and their pathological occlusion might cause ischaemia of this region. These findings support a vascular hypothesis for Crohn's disease and may explain, in part, both the ileal and mesenteric distribution of Crohn's disease ulcers.

(f Clin Pathol 1997;50:1013-1017)

Keywords: Crohn's disease; blood vessels; small intestine; anatomy

A recognised feature of ulcers in Crohn's disease is a propensity to occur along the mesenteric margin of the bowel wall..$^{1-5}$ In one study of Crohn's disease, Yamagata identified longitudinal ulceration along the mesenteric margin in 24 of 26 small bowel resection specimens. ${ }^{5}$ However, only a few reports have explored the underlying basis for this pathological idiosyncracy. In a study examining the vascular changes in inflammatory bowel disease, Brahme and Lindström identified an anatomical association between ileal and colonic Crohn's disease ulcers and the point along the mesenteric margin where arteries break through the muscularis propria, but they did not consider the observation to have any significance. ${ }^{4}$ In a study examining the vascular anatomy of the normal small bowel, Funayama et al identified large anastomotic vessels at the antimesenteric margin of the small intestine that were less abundant along the mesenteric margin $^{6}$; they proposed that in Crohn's disease the mesenteric margin would be more susceptible to episodes of ischaemia and, hence, liable to ulceration.

The involvement of ischaemia as a primary mechanism operating in Crohn's disease has been proposed by Wakefield et al who, on the basis of immunohistological evidence associating granulomas with the vasculature, suggested that ischaemic mucosal injury occurs as a consequence of occlusive granulomatous vasculitis. $^{78}$

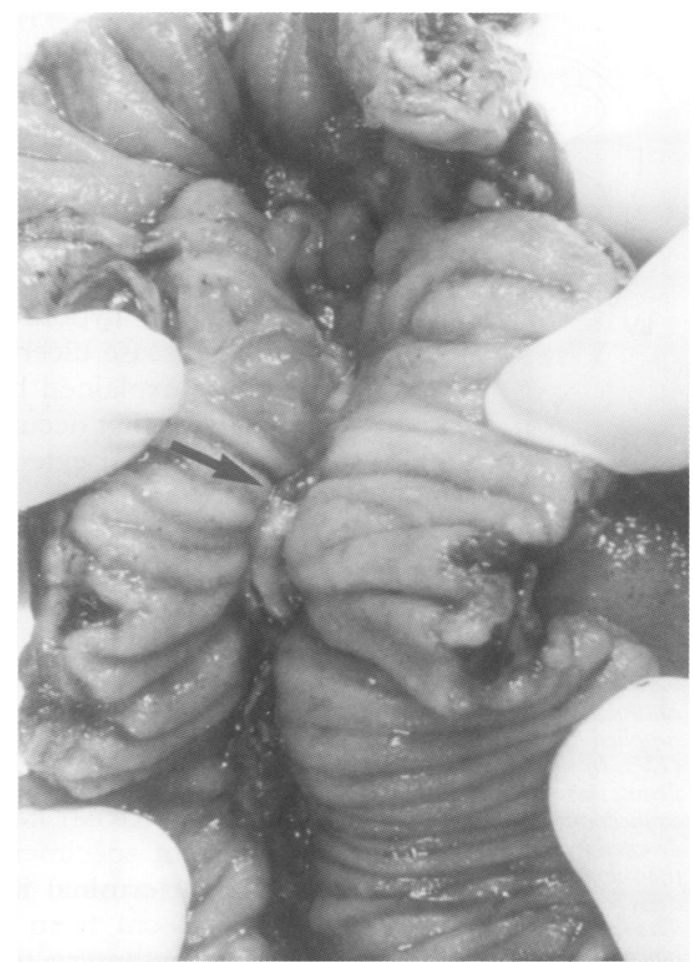

Figure 1 Surgical specimen of ileal Crohn's disease opened along the antimesenteric margin showing a longitudinal ulcer (arrow) along the mesenteric margin. The adjacent mucosa has a normal appearance. 


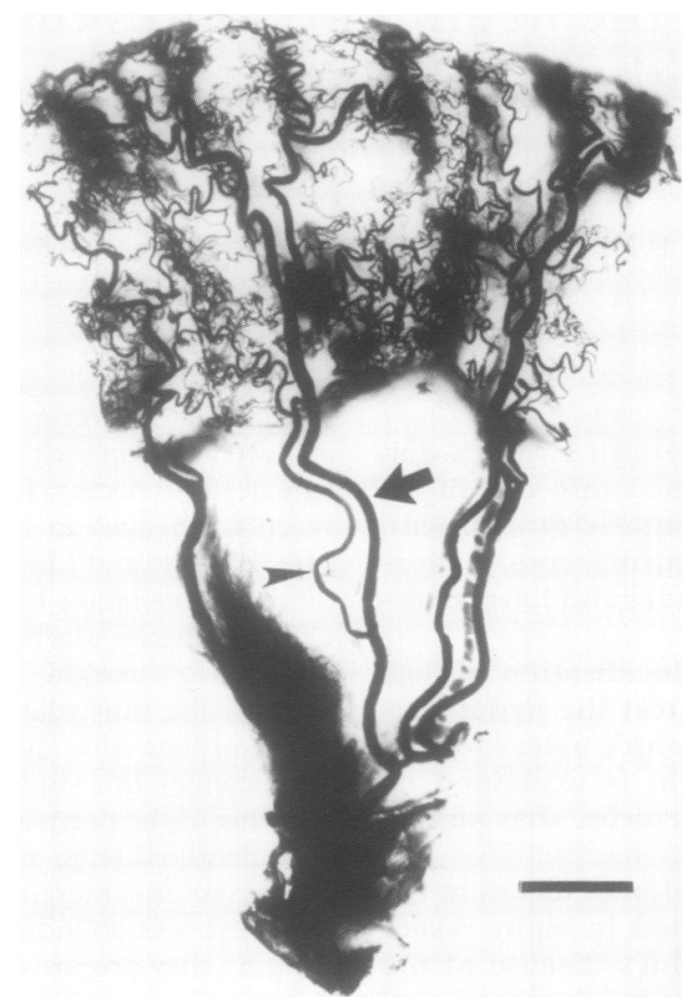

Figure 2 Carbon perfused specimen of human jejunum showing filling of the arterial system. Carbon filled long (arrow) and short vessels (arrowhead) in the mesentery are depicted. $($ Bar $=1 \mathrm{~cm}$.

In an experimental model of inflammatory bowel disease, longitudinal jejunal ulcers induced by indomethacin in the rat also have a preference for the mesenteric margin..$^{9-14}$ Our studies investigating this observation identified ulcer susceptible sites along the mesenteric margin. These sites were supplied by short mesenteric vasa recta that were found to be end arteries. ${ }^{14}$ Because these sites did not have direct communication with the potential compensatory collateral circulation that supplied the rest of the bowel wall, we hypothesised that they would be most sensitive to episodes of ischaemia. ${ }^{14}$

We examined the localisation of ulceration in ileal Crohn's disease resection specimens and explored the hypothesis that predilection of Crohn's disease ulcers for the ileal mesenteric margin is explained by the existence of short end arteries that occur in the human ileum but to a much lesser extent in the jejunum.

\section{Methods}

CROHN'S DISEASE

Figure 4 Schematic to the human small intestine. The mesenteric margin is supplied by short vessels (SV) while the antimesenteric aspect is supplied by longer vessels (LV). In the terminal ileum, there are virtually no connections (at the points denoted by arrows) between the submucosal plexuses derived from $S V$ and $L V$. The two plexuses are, however, well connected in the jejunum.

$A$, antimesenteric aspect

$M$, mesenteric aspect;

$T A$, terminal arcade vessel.
Surgical resection specimens of the terminal ileum from eight confirmed consecutive cases of Crohn's disease (five men and three women; age range, 34-44 years) were examined macroscopically and histologically for mucosal ulceration.

VASCULAR ANATOMY IN POSTMORTEM SPECIMENS Postmortem specimens (20-30 cm in length) of normal terminal ileum and proximal jejunum $(\sim 40 \mathrm{~cm}$ from the pylorus), each with attached mesentery, were dissected from adult cadavers (four men and four women; age range, 55-67 years) in whom no gastrointestinal pathology was found.

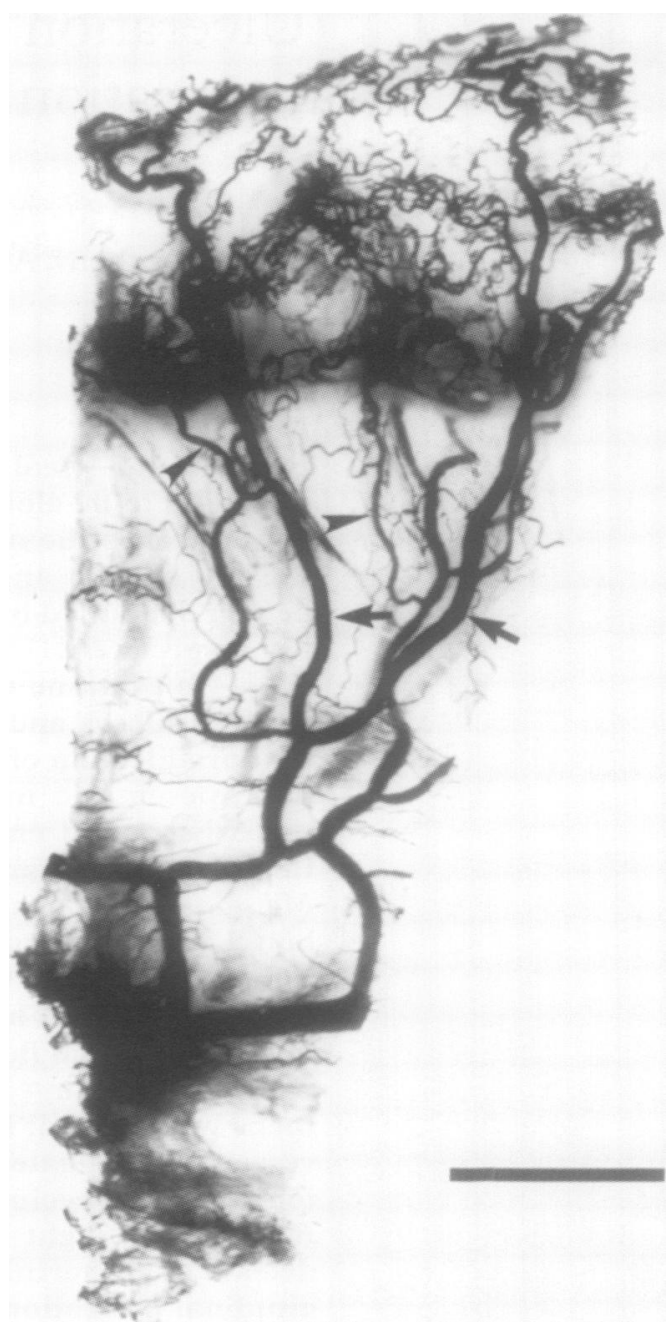

Figure 3 Carbon perfused specimen of ileum showing filling of the arterial system. Carbon filled long vessels (arrows) and short vessels (arrowheads) in the mesentery are depicted. (Bar $=1 \mathrm{~cm}$.)

In each specimen the ends of the bowel and the free edges of the mesentery were clamped and a single mesenteric arcade artery was cannulated with $6 \mathrm{~F}$ intravenous polyethylene tubing. Using a $20 \mathrm{ml}$ syringe, the specimens were arterially perfused with carbon (India) ink until the serosal surface showed maximal colour change. In four of each of the specimens of ileum and jejunum the mesentery was transluminated with a strong light source to highlight the vasculature. In each of these transilluminated specimens isolated single short and long vessels $(-15 \mathrm{~cm}$ apart) were dissected from any mesenteric fat and ligated at their origin before perfusion with ink.

Specimens were fixed (unopened) overnight and solvent cleared to transparency by Spalteholtz's method. ${ }^{15}$ The tissues were stored, examined, and photographed in methyl salicylate. The blood supply of the bowel wall and the submucosal plexus was examined using a laboratory dissecting microscope.

\section{Results}

CROHN'S DISEASE RESECTION SPECIMENS

All eight of the Crohn's disease cases showed longitudinal ulceration along the mesenteric margin. The mucosa adjacent to the ulcers in four of the cases was of normal appearance 


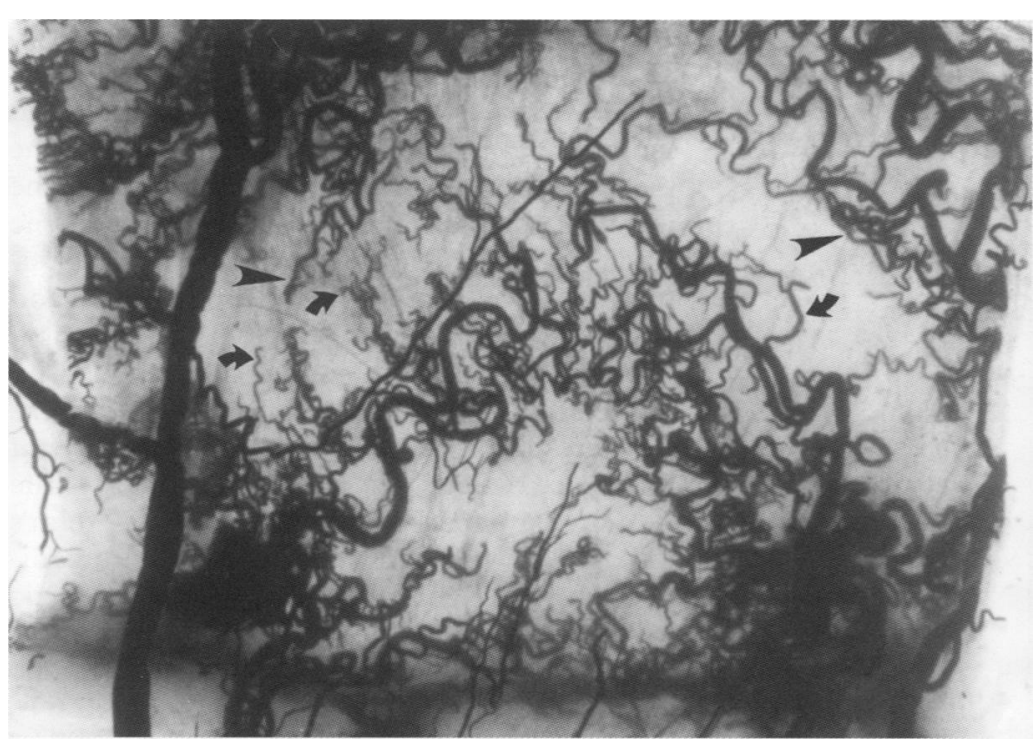

Figure 5 Cleared ileum in which the submucosal vessels derived from short vessels (arrows) have little if any communication with the submucosal plexus derived from the long vessels (arrowheads).

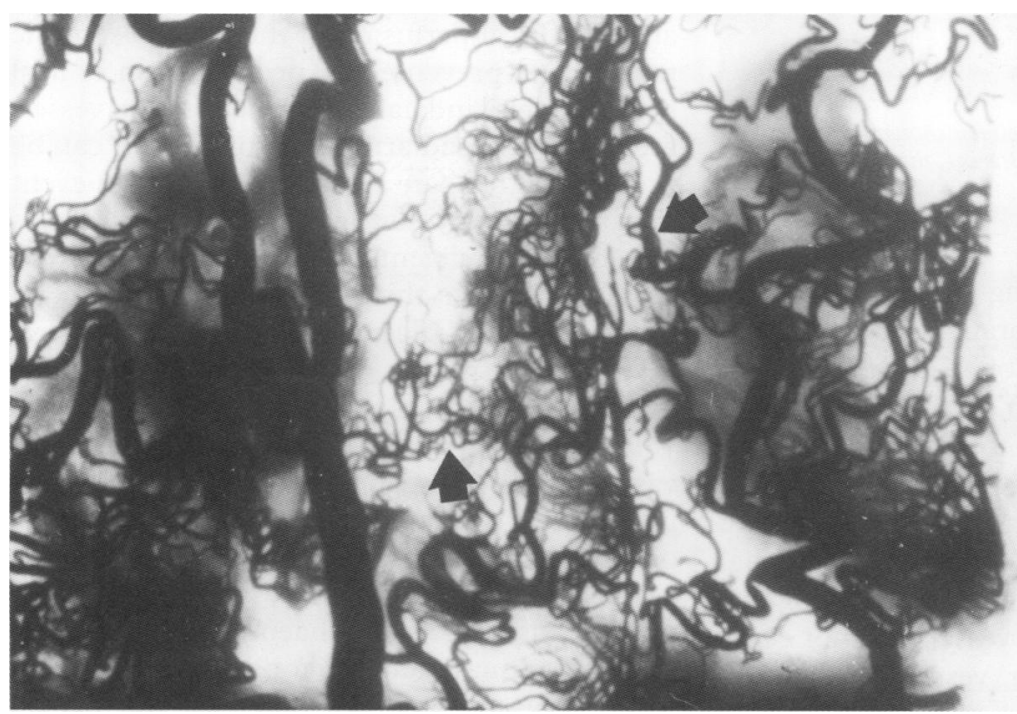

Figure 6 Cleared jejunum showing numerous vessels (arrows) connecting the submucosal plexuses derived from long and short vessels.

(fig 1) while the mucosa in the other four cases showed some degree of oedema. All eight cases showed typical histological features of Crohn's disease that were largely confined to the mesenteric margin of the bowel wall-that is, fissuring ulceration and transmural, acute, and chronic inflammation with granulomas. The granulomatous inflammation underlying the longitudinal ulcers, was associated closely with the mesenteric submucosal vasculature and lymphatics. Granulomas were also evident on the non-ulcerated antimesenteric side of the bowel wall. Overall, the extent of the granulomatous inflammation was most marked in the mesenteric submucosa and extramural mesentery where the largest blood vessels normally resided. The antimesenteric non-ulcerated bowel showed chronic inflammatory cell infiltration and granulomas within the lamina propria.

VASCULAR ANATOMY IN POSTMORTEM SPECIMENS Using a suitable lighting system, it was possible to examine the extramural and submucosal

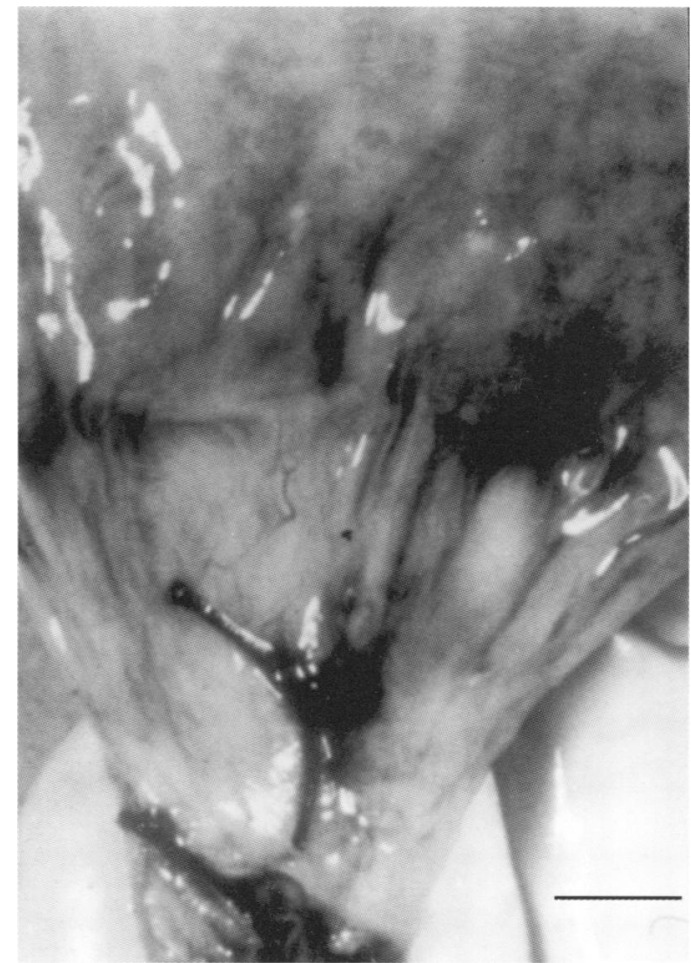

Figure 7 Ligation of a single short vessel in the jejunum did not affect complete filling of the bowel wall. $($ Bar $=1 \mathrm{~cm}$.

vascular anatomy of solvent cleared tissues using a dissecting microscope. The terminal arcade vessels of the mesenteric vasculature gave off, at regular intervals, terminal feeding vessels that supplied alternate sides of the bowel wall (figs 2 and 3). Overall, the vessels supplying the jejunum were of a larger calibre than those supplying the ileum, but at both sites these terminal vessels entered the muscularis propria obliquely at four and eight o'clock (where a transverse plane of the bowel wall is considered to represent a clockface with the mesenteric margin representing six o'clock and the antimesenteric margin representing 12 o'clock; fig 4). In both the ileal and jejunal

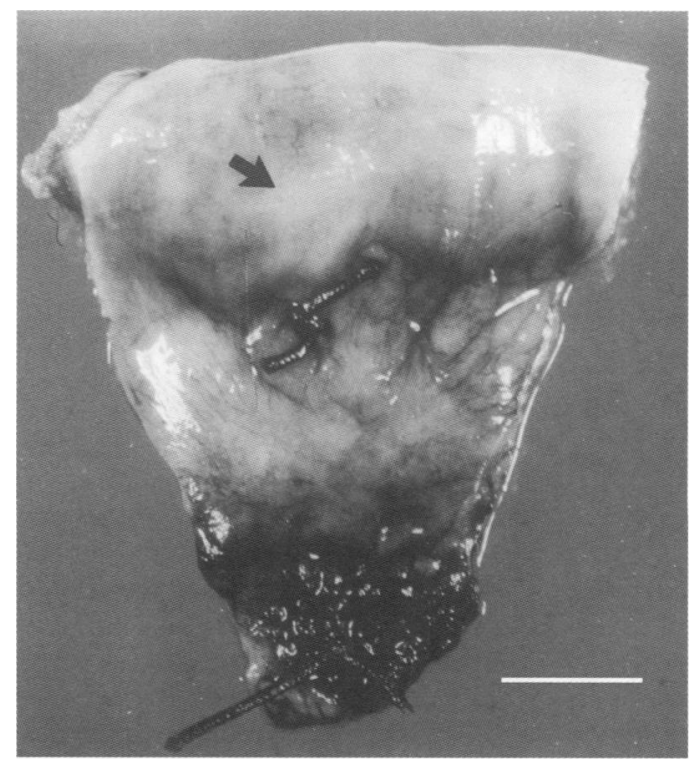

Figure 8 Ligation of a single short vessel in this specimen of ileum prevented local filling (arrow) of the bowel wall along the mesenteric margin. (Bar $=1 \mathrm{~cm}$.) 


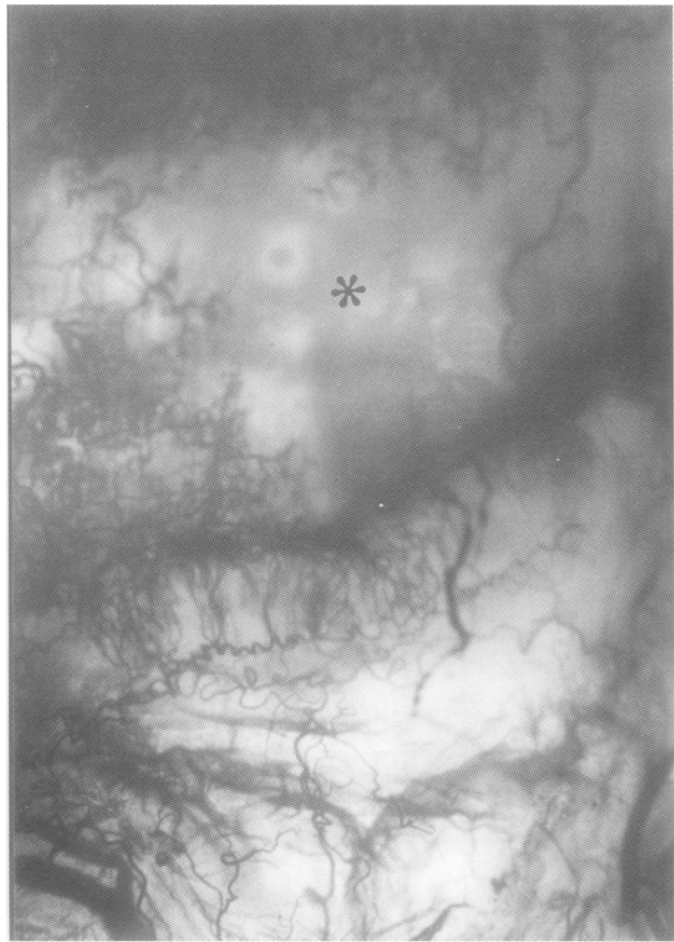

Figure 9 Postclearing of the specimen shown in fig 8. There is an obvious clear area (asterix), the vasculature of which has not filled with ink.

specimens, terminal feeding vessels outside the bowel wall gave off small branches that entered the mesenteric aspect of the bowel wall between five and seven o'clock. These small branches were equivalent to the short supplying vessels identified previously by Griffiths in the human $\operatorname{colon}^{16}$; from here on they are termed "short vessels" while the main terminal branches are termed "long vessels" (fig 4). Individual long and short vessels did not communicate with any other vessel outside the bowel wall.

After entering the bowel wall, short vessels ran a tortuous path and formed a localised submucosal plexus of vessels within the vicinity of the mesenteric margin. In the terminal ileum, short vessels had little or no communication with the main submucosal plexus formed by long vessels that supplied all the bowel wall except the mesenteric margin (fig 5). In contrast, there was extensive communication between these plexuses in the jejunum (fig 6). Long vessels formed a continuous submucosal plexus along the bowel wall along the ileal and jejunal antimesenteric margins.

Ligation of both isolated ileal and jejunal long vessels before carbon ink perfusion did not interfere with the entire filling of the submucosal plexus (fig 7). However, in three of four preparations of the terminal ileum, but in none of the jejunal preparations, ligation of the short vessels resulted in well defined unperfused areas of bowel wall along the mesenteric margin adjacent to the ligated vessel (figs 8 and 9 ), indicating that the intramural plexus of vessels originating from ileal short vessels does not communicate directly with the mural plexus originating from ileal long vessels.

\section{Discussion}

In this study of Crohn's disease of the terminal ileum, we have confirmed the observation that longitudinal ulcers have a predilection for the mesenteric margin. Upon examination of the vascular anatomy of normal specimens of the small intestine, we have confirmed the observation made by Noer ${ }^{17}$ that the mesenteric and antimesenteric margins of both the terminal ileum and proximal jejunum are supplied by short and long supplying arteries, respectively.

There is a clear and potentially important difference between the vascular anatomy of the terminal ileum (a common target for Crohn's disease) and the proximal jejunum (an uncommon target) - that is, the ileal mesenteric submucosal plexus derived from short vessels has virtually no communication with the submucsoal plexus derived from long vessels. This is not the case in the proximal jejunum. This finding, together with the observation that short vessels do not communicate with other arteries either outside or within the bowel wall, and that focal perfusion of the ileal mesenteric margin was prevented by prior ligation of ileal short vessels, indicates that ileal short vessels are effectively end arteries. From this, it can be predicted that an occlusive vascular event occurring at any site along ileal short vessels would cause ischaemic ulceration to dependent areas of mucosa; this prediction could explain the preferental ulceration of the mesenteric margin in Crohn's disease of the terminal ileum.

It has been proposed that granulomatous vasculitis is a pathogenic mechanism operating in Crohn's disease, ${ }^{7}$ so that even a single granulomatous event involving an ileal short vessel (given that the latter is an end artery) would cause mucosal ischaemia and ulceration along the mesenteric margin. An equivalent focus of granulomatous inflammation occurring along a short vessel within the jejunum or within an antimesenteric vessel in the ileum would not cause ischaemia because of the existence of compensating collateral connecting vessels arising from the submucosal plexus.

In microangiographic studies of the intestinal vascular architecture of cadaveric specimens of colon and small intestine, Funayama et al noted that the anastomotic vessels of the submucosal plexus of the antimesenteric margin were far more abundant than those within the mesenteric margin. ${ }^{6}$ They proposed that asynchronous vasoconstriction of vasa recta might induce a longitudinal ischaemic area on the mesenteric side but, hitherto, there has been no evidence to support such a hypothesis. Blood flow through short vessels could also be compromised during periods of intense spasm of the muscularis propria, which might compress vessels of smaller calibre more easily than those of larger calibre. It has been shown that electrical field stimulated contractions of the rat stomach can reduce or stop gastric mucosal blood flow, ${ }^{18}$ and spasmolytic agents can protect against experimental nonsteroidal antiinflammatory drug induced gastrointestinal ulceration thought to arise, at least in part, as a 
consequence of gastrointestinal smooth muscle contraction. ${ }^{19} 20$

The idea that muscular compression of gastrointestinal end arteries would cause mucosal ischaemia at a susceptible anatomical site is not new. Piasecki found that the propensity of human peptic ulceration to occur along the lesser curvature of the gastric antrum was related directly to the increased prevalence of "mucosal end arteries of extramural origin" at that site. ${ }^{21} \mathrm{He}$ proposed that muscular compression or vasoconstriction of such functional end arteries during periods of stress or experimental ligation would result in mucosal ischaemia and ulceration. ${ }^{22}{ }^{23}$ Although the pathogenesis of Helicobacter pylori induced peptic ulceration is unclear, inflammatory mediators acting on gastric smooth muscle and vascular tone may be important.

In conclusion, Crohn's disease ulcers of the terminal ileum occur preferentially along the mesenteric margin. In the terminal ileum, but not in the proximal jejunum, the mesenteric margin is supplied by short vessels that are effectively end arteries and supply mucosa that may be susceptible to ischaemia. These findings help to explain the preferential distribution of Crohn's ulcers along the mesenteric margin and they may explain, in part, the susceptibility of the terminal ileum to this chronic gastrointestinal disorder; they support a vascular hypothesis for the pathogenesis of Crohn's disease. A part of this study was supported by GlaxoWellcome Research
and Development, UK. The authors would like to thank $\mathrm{Mr}$ and Development, UK. The authors would like to thank Mr Chris Thrasivoulou (Department of Anatomy, Royal Free Hos-
pital School of Medicine) for his technical assistance in the pital School of Medicine) for his
preparation of the cleared tissues.

1 Crohn BB, Ginsberg L, Oppenheimer G. Regional ileitis. A

pathological and clinical entity. $\mathcal{F} A M A$ 1932;99:1323-9.
van Patter WN, Bargen JA, Dockerty MB, Feldman WH,

van Patter WN, Bargen JA, Dockerty MB, Feldman WH, Mayo C

3 Warren S, Sommers SC. Pathology of regional ileitis and ulcerative colitis. $¥ A M A$ 1954;154:189-93.

4 Brahme F, Lindström C. A comparative radiographic and pathological study of intestinal architecture in Crohn's disease and in ulcerative colitis. Gut 1970;11:928-40.

5 Yamagata S. Crohn's disease in Japan: report of the Japanese committee for Crohn's disease. In: Lee ECG, ed Crohn's workshop - a global assessment of Crohn's disease. London: HM Publishers, 1981:136-44

6 Funayama Y, Sasaki I, Naito Y, Kamiyama K, Fuckushima $\mathrm{K}$, Sato T. Micro-angiographic studies of intestinal vasoarchitecture with special reference to ulcerogenesis in Crohn's disease. $7 \exists_{p n}$ Soc Coloproctol 1987;40:839-44.

7 Wakefield AJ, Sankey EA, Dhillon AP, Sawyer AM, More L, Sim R, et al. Granulomatous vascultis in Crohn's disease. Gastroenterology 1991;100:1279-87.

8 Wakefield AJ, Dhillon AP, Rowles PM, Sawyer AM, Pittilo RM, Lewis AAM, et al. Pathogenesis of Crohn's disease: multifocal gastrointestinal infarction. Lancet 1989;ii:105762.

9 Kent TH, Cardelli RM, Stamler FW. Small intestinal ulcers and intestinal flora in rats given indomethacin. Am $\mathcal{f}$ Pathol and intestinal flor

10 Wax J, Clinger WA, Varner P, Bass P, Winder CV. Relationship of the enterohepatic cycle to ulcerogenesis in the rat small bowel with flufenamic acid. Gastroenterology 1970;58: 772-80.

11 Anthony A, Dhillon AP, Nygard G, Hudson M, Piasecki C, Strong $P$, et al. Early histological features of small intestinal injury induced by indomethacin. Aliment Pharmacol Ther injury induced

12 Anthony A, Dhillon AP, Thrasivoulou C, Pounder RE, Wakefield AJ. Pre-ulcerative villous contraction and microvascular occlusion induced by indomethacin in the rat jejunum: A detailed morphological study. Aliment Pharmacol Ther 1995;9:605-14.

13 Matsumoto T, Iida $M$, Nakamura S, Hizawa $K$, Kuroki $F$, Fujishima $M$. Preventive effect of immunosuppresive agents against indomethacin-induced small intestinal ulcers in rats. Dig Dis Sci 1994;39:787-95.

14 Anthony A, Dhillon AP, Pounder RE, Wakefield AJ. Vascular anatomy defines sites of indomethacin-induced jejunal ulceration along the mesenteric margin. Gut 1997;41:76370 .

15 Spalteholtz H. Ruber das durchsidutigmachen von menschlichen und tierischen preparaten. S Histzol Leipzig 1914.

16 Griffiths JD. Extramural and intramural blood-supply of colon. BMF 1961;i:323-6.

17 Noer RJ. The blood vessels of the jeunum and ileum: a comparative study of man and certain laboratory animals. $\mathrm{Am} \mathcal{F}$ Anat 1943:73:293-334.

18 Livingston EH, Howard TJ, Garrick TR, Passaro EP, Guth $\mathrm{PH}$. Strong gastric contractions cause mucosal ischaemia. Am f Physiol 1991;260:G524-30.

19 Takeuchi K, Okada M, Ebara S, Osano H. Increased microvascular permiability and lesion formation during gastric hypermotility caused by indomethacin and 2-deoxy-Dglucose in the rat. 7 Clin Gastroenterol 1990;12(suppl 1):S76-84.

20 Anthony A, Bahl AK, Dhillon AP, Oakley IA, Piasecki C, Spraggs CF, et al. The beta ${ }_{3}$-adrenoceptor agonist CL316243 prevents indomethacin-induced jejunal ulceration in the rat by reversing early villous shortening. $\mathcal{F}$ Pathol 1996;179:340-6.

21 Piasecki C. Blood supply to the human gastroduodenal mucosa with special reference to ulcer bearing areas. $\mathcal{F}$ Anat 1974:118:295-335.

22 Piasecki C. Evidence for an infarctive pathogenesis of acute and chronic gastroduodenal ulceration. $\mathcal{f}$ Physiol Pharmacol 1992;43:99-112.

23 Piasecki C, Thrasivoulou C, Rahim A. Ulcers produced by ligation of individual gastric mucosal arteries in the guinea pig. Gastroenterology 1989;97:1121-9. 